\title{
Resonant Diaphragm Liquid Density Sensor
}

\author{
Yuxiao Zhang ${ }^{1, a}$, Xianquan Zeng ${ }^{1, b}$, Xinjie Wang ${ }^{1, c}$ \\ ${ }^{1}$ Department of Electromechanical Science and Engineering, Zhengzhou University of Light \\ Industry, Dongfeng Road No.5, Zhengzhou, Henan, China \\ ajasonzyx@126.com, bzengxianqun012345@163.com, cweibo1991@126.com
}

Keywords: Density sensor; Resonance; Residual stress;

\begin{abstract}
This paper designs a resonant diaphragm liquid density sensor. From the reality, a resonant diaphragm is designed and manufactured. Processing technology on resonant diaphragm is studied and improved through compensating elastic error, and eliminating residual stress. Incentive coil is designed using the eddy current effect.
\end{abstract}

\section{Introduction}

Because liquid density measurement has great significance in the production practice, liquid density sensor is developing very quickly recent decades. [1] and [2] introduced two different liquid density sensor with vibratory tube, one kind was straight tube type, another kind was u-shaped tube type, but they shared the same basic working principle. As bend tube had more resistance than straight tube when liquid flowed, it lost more pressure than straight tube. Vibratory tube liquid density sensor would have some design problems too difficult to overcome, such as end load, pressure. When the fluid flowed through vibration tube, stress acted on the pipe for pressure is imbalance, which led to rigidity changing. [3] designed a vibratory tube density sensor to measure trace liquid. It used modern micro-processing technology to bond two pieces of silicon together to make a tiny silicon tube. And density of the liquid could be gained by impelling the tube with static electricity and detecting the change of its resonant frequency with optical method. [4], [5] and [6] introduced another resonant liquid density sensor naming resonant barrels liquid density sensor. Resonant barrels liquid density sensor used the relationship between mechanical vibratory frequency and liquid density. [7] introduced a tuning fork liquid density sensor which used inherent frequency's changing of the tuning with low quality in liquid to measure density.

\section{Design Resonant Diaphragm}

In order to guarantee the processed liquid density sensor meeting the design requirements, resonant diaphragm processing must meet the following demands.

Diaphragm smooth finish should be as good as possible.

Holes on the two sides of the diaphragm should have the same circular degree.

Holes on the two sides of the diaphragm should be coaxial.

Planes on the two sides of the diaphragm should be flat and paralleled. 


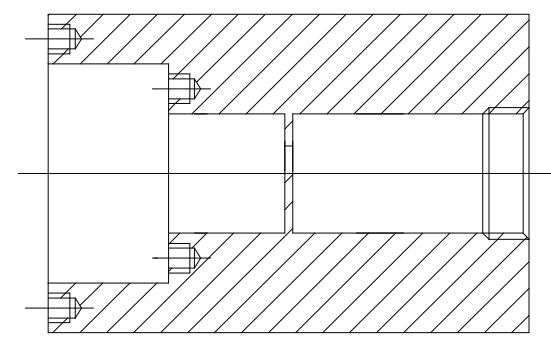

Fig.1 Shematic Diagr

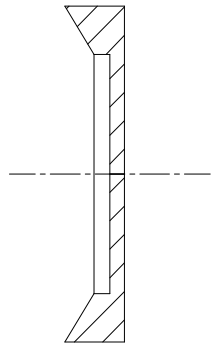

Fig.2 Vibratory Diaphragm

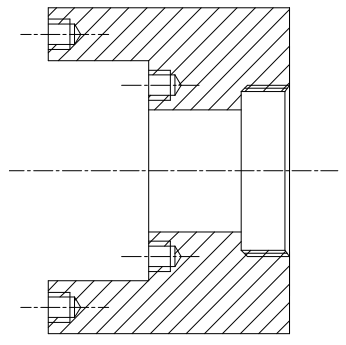

Fig.3 Installation Circuit Board

Fig.1 shows a shematic diagram designed for density sensor. It is also the most ideal structure. This structure is very compact for integrating sensitive component and installation circuit board. And liquid undulation has very small impact on the sensor. In actual processing project, however, common processing technology is unable to realize the structure.

According to the existing processing technology and analyzing again, a new sensor structure is designed, which meets the design demands and can be processed successfully. Fig.2 shows vibratory diaphragm structure. Fig.3 shows a structure used to install relevant circuits called substrate. Diaphragm and substrate are connected with each other by screw structure.

\section{Research on Processing Technology}

Elastic deformation error compensation technology. During the machining process, cutter and workpiece may cause larger elastic deformation, due to cutting force. When analyzing the problem on controlling and compensating elastic deformation error, workpiece can be regarded as having ideal rigidity and surface machining error is only concerned with cutter deformation if the workpiece rigidity far outweigh the tool rigidity. For the common thin-walled parts, it must be taken into consideration that potentially obvious machining deformation workpiece has and the complex coupling effect, which has with cutter deformation. In the actual operation, circular error compensation scheme is adopted.

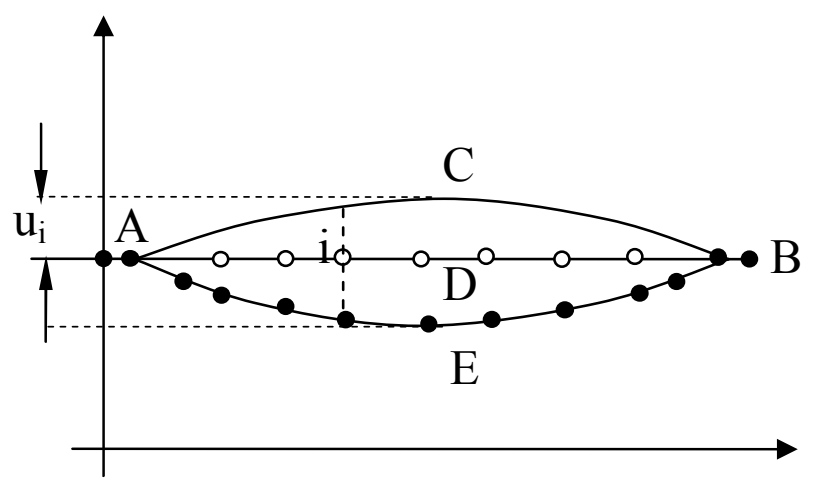

Fig.4 Machining Deformation Error Compensation

In the Fig. 4, the curve ACB shows predicted distribution regularity on the surface of workpiece. The curve ADB shows nominal cutter path exported by CAD/CAM system. The curve AEB shows corrected cutter path. On cutter point $\mathrm{i}$, there is a relationship among corrected cutter position $\mathrm{X}_{\mathrm{e}}$, nominal cutter position $\mathrm{X}_{\mathrm{di}}$ and machining deformation $\mathrm{U}_{\mathrm{i}}$. The relationship can be expressed as

$$
\mathrm{X}_{\mathrm{ei}}=\mathrm{X}_{\mathrm{di}}+\mathrm{U}_{\mathrm{i}}
$$


According to offline compensation principle, only can the machining deformation be predicted, machining errors caused by workpiece and cutter deformation can be eliminated effectively by correcting every cutter point's CNC code.

Residual stress and its processing method. Either turning or milling would induce residual stress in workpiece surface layer during machining process. The residual stress state affects parts surface fatigue crack nucleating and extending, which would reduce fatigue life of parts very much.

Residual stress has great impact on the vibratory frequency of resonant diaphragm. At present, natural aging method and artificial aging method are used to eliminate residual stress. Artificial aging method includes heat ageing, knock ageing, vibration aging, ultrasonic impact aging, explosion aging. This paper adopt natural aging method, heat ageing and vibration aging to process vibratory diaphragm.

Fig. 5 shows 3 kinds of vibratory diaphragm. Diaphragm (a) has been processed with vibration aging and heat ageing, and the surface has changed. Diaphragm (b) has been processed with vibration aging. Diaphragm (c) has not been processed with residual stress elimination and surface processing quality has changed.

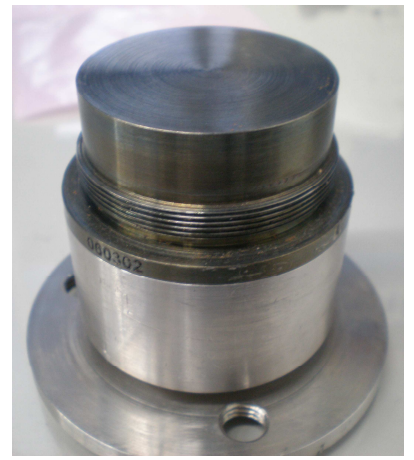

(a)

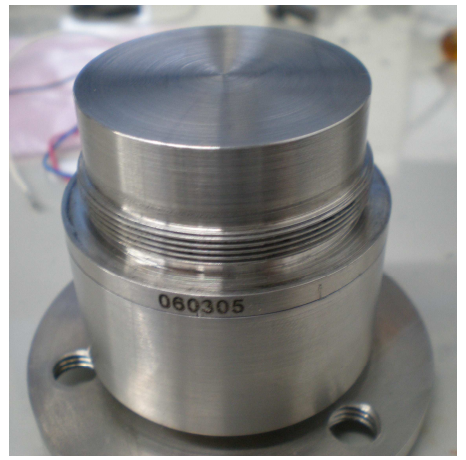

(b)

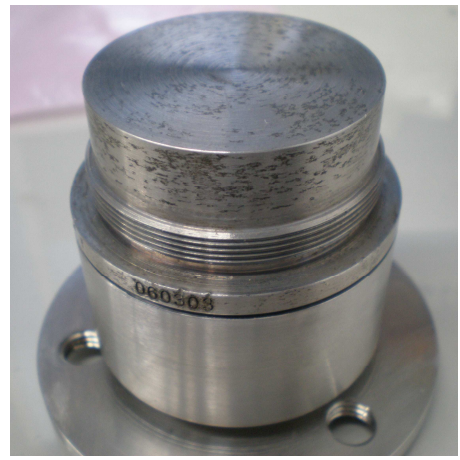

(c)

Fig. 5 Vibratory Diaphragm

Design incentive coil. As shown in Fig. 6, incentive coil is placed in the neighborhood of diaphragm metal conductor but does not connect with each other. When the high frequency alternating current $i_{1}$ passes the incentive coil, a alternating magnetic field $\phi_{1}$ is being generated surrounding the coil. Alternating magnetic field $\phi_{1}$ generates a eddy current $i_{2}$ on the surface of nearby metallic conductor and another alternating magnetic field $\phi_{2} . \phi_{1}$ and $\phi_{2}$ share the same changing frequency but their directions is opposite to each other. There exists mutual effect between incentive coil and diaphragm because of magnetic effect. And this is the explanation for incentive coil exciting diaphragm.

Incentive coil is a hollow coil. If the turns number is $\mathrm{N}$, the magnetic field it generated is equal to the sum of the magnetic field generated by $\mathrm{N}$ single circle coil. Along the radial direction, magnetic induction increases near the coil and obtains maximum at the position 1/3 external diameter to center. Then magnetic induction diminishes quickly and its direction reverses approach to external diameter. In the coil distance, magnetic induction decreases monotonely. The farther a position is away from the coil, the slower it decreases. Along the axial direction, the magnetic induction decreases exponentially. 
According to above analysis, the incentive coil is designed into two parts. One is coil, and the other is adjustable lever fixing the incentive coil. Fig. 7 is the installation drawing for incentive coil and adjustable lever. Incentive coil and adjustable lever can be fixed together with screws.

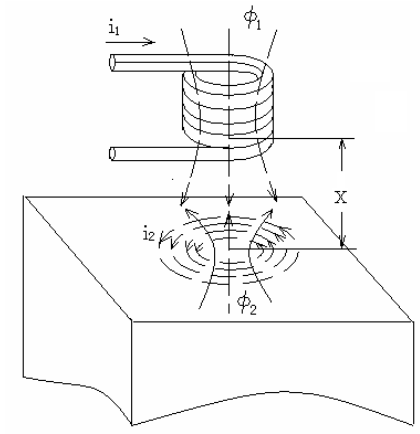

Fig. 6 Eddy Current Effect

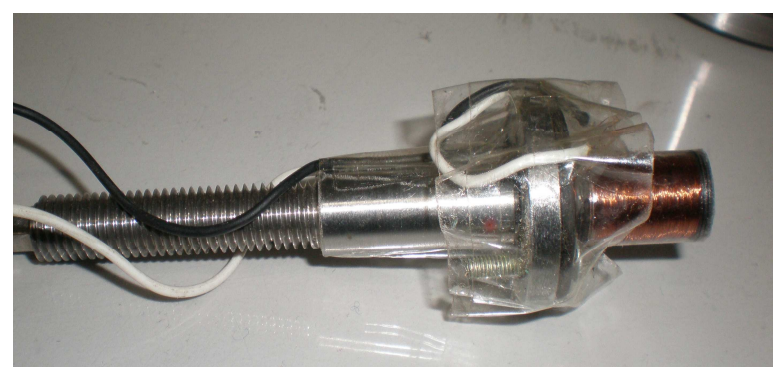

Fig.7 Installation Drawing

\section{Conclusion}

The paper has designed the most critical part resonant diaphragm of the liquid density sensor. Analysing the influences on resonant diaphragm, solutions has been found to elastic deformation and residual stress. So the process quality has been improved. Meanwhile, a incentive coil has been developed to excite resonant diaphragm.

\section{Acknowledgements}

This work was financially supported by the National Natural Science Foundation of China (50875246).

\section{References}

[1] Baohai Tan, Xiaodong Ju: Well Logging Technology. Vol.28 (2004), p. 41-43

[2] Xinhua Wei, Jiawei Gong, Guyuan Yu and Jinxing Wang: Transactions of The Chinese Society of Agricultural Machinery. Vol.32 (2001), p.89-93

[3] M. Najmzadeh, S. Haasl and P. Enoksson: Micromechanics and Microengineering.Vol. 17 (2007), p. 1657

[4] Weidong Chen, Yuming Fan: Elettronic Measurement Technology. Vol.29 (2006), p. 175-177

[5] Jieru Xie, Shenfang Yuan: Manufacturing Information Engineering of China. Vol. 35 (2005), p. 34-36

[6] Xiuwu Sui, Wei Yao: Chinese Journal of Sensors and Actuators. Vol. 18 (2005), p. 394-397

[7] Deqiu Lin: Automation in Petro-chemical Industry. No.2 (1998), p. 52-55 